\title{
Simple training tricks for mastering and taming bypass procedures in neurosurgery
}

\section{Hafez, A.}

2017

Hafez , A , Raj , R , Lawton , M \& Niemelä , M 2017 , ' Simple training tricks for mastering and taming bypass procedures in neurosurgery ' , Surgical Neurology International , vol. 8 , no. 1 , 295 .https://doi.org/10.4103/sni.sni_322_17

http://hdl.handle.net/10138/300033

https://doi.org/10.4103/sni.sni_322_17

cc_by_nc_sa

publishedVersion

Downloaded from Helda, University of Helsinki institutional repository.

This is an electronic reprint of the original article.

This reprint may differ from the original in pagination and typographic detail.

Please cite the original version. 


\title{
Original Article
}

\section{Simple training tricks for mastering and taming bypass procedures in neurosurgery}

\author{
Ahmad Hafez, Rahul Raj, Michael T. Lawton¹, Mika Niemelä \\ Department of Neurosurgery, University of Helsinki and Helsinki University Hospital, Helsinki, Finland, ${ }^{1}$ Department of Neurosurgery, Barrow Neurological \\ Institute, Phoenix, Arizona, USA \\ E-mail: *Ahmad Hafez - ext-ahmad.hafez@hus.fi; Rahul Raj - rahul.raj@hus.fi; MichaelT. Lawton - Michael.Lawton@BarrowBrainandSpine.com; \\ Mika Niemelä - mika.niemela@hus.fi \\ *Corresponding author \\ Received: 31 August $17 \quad$ Accepted: 06 October $17 \quad$ Published: 06 December 17
}

\begin{abstract}
Background: Neurosurgeons devoted to bypass neurosurgery or revascularization neurosurgery are becoming scarcer. From a practical point of view, "bypass neurosurgeons" are anastomosis makers, vessels technicians, and time-racing repairers of vessel walls. This requires understanding the key features and hidden tricks of bypass surgery. The goal of this paper is to provide simple and inexpensive tricks for taming the art of bypass neurosurgery. Most of these tricks and materials described can be borrowed, donated, or purchased inexpensively.

Methods: We performed a review of relevant training materials and recorded videos for training bypass procedures for 3 years between June 2014 and July 2017. In total, 1,300 training bypass procedures were performed, of which 200 procedures were chosen for this paper.

Results: A training laboratory bypass procedures is required to enable a neurosurgeon to develop the necessary skills. The important skills for training bypass procedures gained through meticulous practice to be as reflexes are coordination, speed, agility, flexibility, and reaction time. Bypassing requires synchronization between the surgeon's gross movements, fine motoric skills, and mental strength. The suturing rhythm must be timed in a brain-body-hand fashion.

Conclusion: Bypass-training is a critical part of neurosurgical training and not for a selected few. Diligent and meticulous training can enable every neurosurgeon to tame the art of bypass neurosurgery. This requires understanding the key features and hidden tricks of bypass surgery, as well as uncountable hours of training. In bypass neurosurgery, quality and time goes hand in hand.
\end{abstract}

Key Words: Bypass neurosurgery, simple training, skill

Videos available on: www.surgicalneurologyint. com

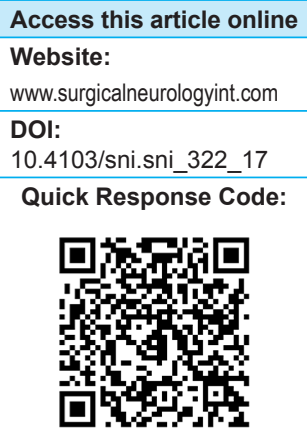

This is an open access article distributed under the terms of the Creative Commons Attribution-NonCommercial-ShareAlike 3.0 License, which allows others to remix, tweak, and build upon the work non-commercially, as long as the author is credited and the new creations are licensed under the identical terms.

For reprints contact: reprints@medknow.com 


\section{INTRODUCTION}

Neurosurgery is considered to be a highly demanding field with time consuming and intensive training. ${ }^{[4,13]}$ Vascular and bypass neurosurgery may be considered even more demanding, requiring meticulous and long training hours. ${ }^{[1,8,15,18,19]}$ The need for vascular neurosurgeons will probably decrease in the future with the changes in the landscape of neurovascular diseases in combination with advances in endovascular techniques. ${ }^{[5,9,12]}$ Still, the need for high level of bypass neurosurgeons will continue to solve many critical vascular pathologies, such as large fusiform aneurysm, moyamoya disease, some tumor cases, selective cases of Transient Ischemic Attack (TIA) patients, and others. The decrease in neurovascular procedures impose challenges in the training of future neurovascular surgeons. Recent techniques such as simulation and virtual reality have been developed to facilitate neurosurgical training. ${ }^{[2,3,6,10,11,14,17,19]}$ Yet, the reliability of these techniques in producing better neurosurgeons is still to be verified. Moreover, the availability of these novel training methods is limited. Neurovascular surgical procedures have long learning curves. This is complicated by the fact that few resident programs offer the opportunity to perform bypass surgery for residents. For example, in the U.S. and Europe, there are currently no standards for training neurosurgical residents regarding bypass surgery.

Bypass procedures technically involve several phases such as preparing the donor vessel, harvesting a graft, preparing the recipient vessel, and microanastomosis. Training for bypass surgery consists of two main parts - eye-hand coordination and knowledge of microsuturing tricks. Eye-hand coordination enables fine motor skills and control of the microinstruments, which are the foundation of microsuturing. ${ }^{[6,7,16,19]}$ To be able to flexibly control the instruments at all times is imperative for successful bypass procedures. Microsuturing demands interchangeable smooth closing and opening pressures of the microinstruments, even in suboptimal positions. Thus, microsuturing for bypass neurosurgeons should come as a reflex not requiring concentration. This can only be achieved by repetitious and meticulous laboratory training. Furthermore, the neurosurgeon must remain calm during the whole procedure even when facing crisis or unstable situations. During practicing, the ability to eliminate all unnecessary movements to minimize time is a key factor before moving on to real surgery.

The goal of this paper is to provide simple and inexpensive tricks for taming the art of bypass neurosurgery. Most of these tricks and materials described can be borrowed, donated, or purchased inexpensively.

\section{MATERIALS AND METHODS}

We performed a review of relevant training materials and recorded videos for training bypass procedures performed by the first author (AH) for 3 years between June 2014 and July 2017. In total, 1,300 training bypass procedures were performed, of which 200 procedures were chosen for this paper. The 200 chosen procedures were all end-to-side procedures performed in $3 \mathrm{~cm}$ depth, simulating the most common superficial temporal artery to medial cerebral artery (STA-MCA) bypass procedure. Two types of materials were used: in 100, a 1-mm chicken wing vessel was used, and in 100, a $1-\mathrm{mm}$ wet plastic tube was used. The recipient vessel was prepared the same way for all procedures [Video 1]. For each material, 1:1 were interrupted microsuturing [Video 2] and uninterrupted microsuturing [Video 3]. The bypass setup is shown in Figure 1. The input and experience of two senior neurosurgeons (ML, MN) were considered when analyzing the training videos.

\section{RESULTS AND DISCUSSION}

\section{Phases of the micro-suture}

The bypass neurosurgeon's goal is to develop the highest possible procedure velocity. For experienced bypass neurosurgeons, the warm-up stage is short, and may reach optimum velocity after a couple of sutures. Every microsuture consists of two major phases - the penetrating (support) phase and the knotting (recovery) phase. The neurosurgeon's speed is produced during both phases.

In the penetration phase, the needle driving hand applies gentle force against the wall of the vessel from a vertical orientation. Force is applied for a short time and the vessel wall is penetrated. To maintain flexible velocity, the neurosurgeon must be able to effectively apply suitable

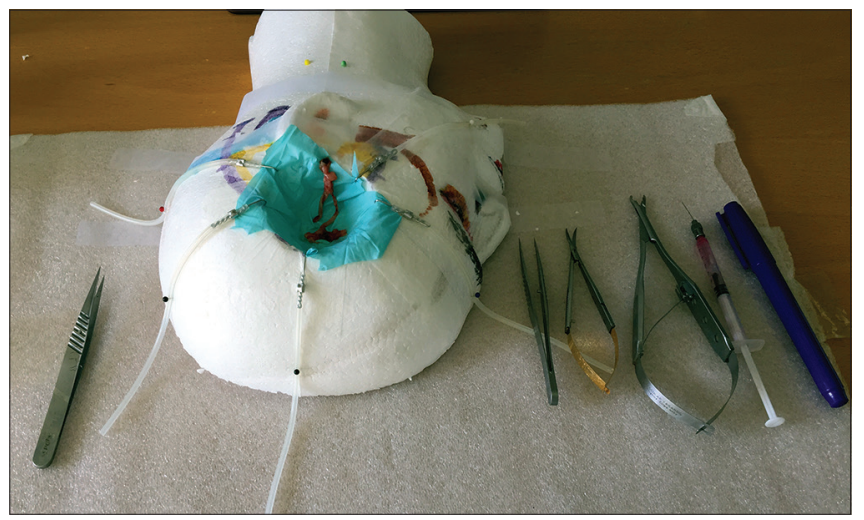

Figure I:The bypass training setup and basic microtools. On the left side a pair of forceps, from the far right side a marker pen, a syringe, a clip holder, a pair of scissors, and a pair forceps. In the middle, a self-made model of a human head by Styrofoam. In the middle of the head (frontotemporal junction) and plastic tube vessel mimicking the superior temporal artery 
force during this short time period. After the penetration phase comes the knotting phase. The microsuture velocity is the product of these two phases. Further, our experience is that the two phases interact. If one phase gets slower the other will also slow down and vice versa.

\section{Steps of the bypass procedure}

Only three main instruments are needed in a majority of bypass procedures, two forceps and a scissor. Our experience and practicing in the lab supports self-handling of scissor by placing it as close as possible to the right hand of a right-handed neurosurgeon. The whole bypass procedure can be compared to a running performance. First comes the takeoff, followed by acceleration, and maintenance of maximum speed before decelerating and finishing the race (or bypass).

1. The takeoff phase at two points start (most time-consuming phase for most neurosurgeons)

2. The acceleration phase

3. The phase of maximum (constant speed)

4. The deceleration phase (checking time).

5. The finish.

During the takeoff phase the donor vessel is stitched to the recipient vessel at 12 and 6 o'clock. This requires a wide range of motion and different needle directions. This is often the most time-consuming phase. Following the takeoff phase, the acceleration phase starts with continuous repetition of stitches along the sides of the vessels. During the acceleration phase each stich should be placed faster and faster. After a few stitches, the surgeon reaches his/her maximum speed. When the stitches are coming to an end the surgeon usually has to decelerate to check the patency of the stitches before ending the suturing. Moving to the other side of the vessel should not disturb the procedure. In reality, the surgeon tends to move the chair, the microscope, the instruments, as we have the feeling of working in

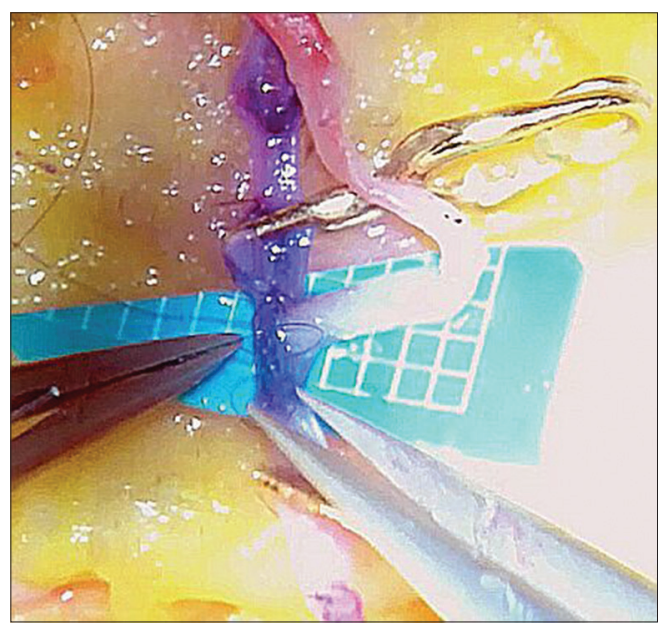

Figure 2: Uninterrupted suturing with tightening of the stitches at the end.We find this technique not as good as tightening after every single stitch. The technique puts the thread at a risk of being accidentally cut before they are tightened different sides of the "wall," which is not true. For right-handed neurosurgeons, putting the upper endpoint stitch at 11 o'clock may be preferable and for left-handed neurosurgeons putting the upper endpoint stitch at 1 o'clock may be preferable.

For surgeons who perform uninterrupted stitching, we have found it preferable to tighten the thread after each stich instead of loosely suturing the whole vessel side and tightening the stitches at the end [Figure 2]. For interrupted microsuturing, the needle should preferably always be kept in the view of the microscope, without the need of zooming out. Zooming out and in helps the surgeon to tighten the stitch under low magnification and handle the thread in an easier manner, making the transition for the next knot easier. A foot pedal controlling the zoom function is helpful.

Skills needed for taming bypass procedures Coordination: The skill of the neurosurgeon performing high-speeded movements requires nervous system control, which is the most critical aspect for effective bypassing.

Speed: This is obviously an important factor. Speed is tied to coordination, the ability to move the finger and hands at high velocities, and express precision and accuracy through those movements to proceed at high velocities.

Agility: The ability to change the hands and fingers position efficiently, which requires the integration of isolated small movement skills. It involves the combination of coordination, speed, reflexes, strength control, and stress endurance.

Flexibility: Bypass-mastering necessities high degree of flexibility in all parts of body, not only fingers and hands but also the whole upper-body, neck, and head.

Reaction time: "Nano" reaction time is a critical to perform a bypass in less than 20 minutes. Usually the time is lost after making the knot and getting a new grip of the needle.

These characteristics can be developed depending on the training program. The training program should be directed towards the development of all mentioned skills. A training laboratory giving the opportunity to train bypass procedures is required to enable the neurosurgeon to develop the skills necessary. Easily and cheaply available plastic tubes and chicken wings are suitable for training.

Bypass tricks

Below some tricks for the surgeon interested in bypass procedures:

- Hard sport may not be suitable the day of surgery. Fine tremor after vigorous sporting (e.g., weight lifting, boxing) may cause fine motor tremor several hours afterwards 
- Prior to the operation, the neurosurgeon should avoid muscle exertion. For example, try to avoid straining yourself by holding the patient's head for prolonged times when placing the patient's head into the right position in the operation room

- Be relaxed and sit comfortably. Your favorite music may help you relax

- The position of the surgeon is important and should be coordinated with the microscope. The lens of the microscope should be vertical with the operation field. Thus, mild flexion of the neck is often required to optimize the surgeon's position. This combined with elbows gently squeezing against the waist to neutralize big joint movements gives you the optimal position for microsuturing

- Always keep the needle and the thread on a focused level in the microscope view

- The needle is held at a two-third distance from the tip

- Forceps should be leaner than the needle holder

- When making the knot, move only the forceps. Keep the needle holder still (unnecessary movement)

- Pull the first knot to your side, the second to the opposite, and the third again to you. When holding the end to cut the remainder of the thread, keep the thread between the grasp of the forceps. In this manner, the thread is under your control and it is easier to start the second knot [Figure 3]

- Keep the angle between yourself and the suturing direction close to $180^{\circ}$. This allows you to put the suturing axis at 11-12 o'clock, or at 1 o'clock for left-handed neurosurgeons

- Putting the contralateral edge of the donor vessel inside the lumen of the recipient vessel is a practical trick to avoid penetrating the contralateral edge of the receiver [Figure 4]

- A clean surgical field is important. Yet, exaggerated water flushing may move your needle and make you work under a water-blurred vision for some time [Figure 5]

- Keep the end piece of the thread after penetrating the vessels as short as possible before starting the knot. This helps you find the thread's end piece quickly, saving a lot of unnecessary hand movements [Figure 6].

\section{Training suggestions}

- Laying the foundation for eye-hand coordination and establish a microsuture routine. Perform microsutures for a minimum of 1 hour per day for at least 3 months. Use 7-0 or 8-0 thread. Do not focus on speed or time. The speed will be developed automatically

- Improve the microsuturing technique by clocking the time without placing too much emphasis on it. Use 9-0 thread. Putting a tube in the recipient vessel is

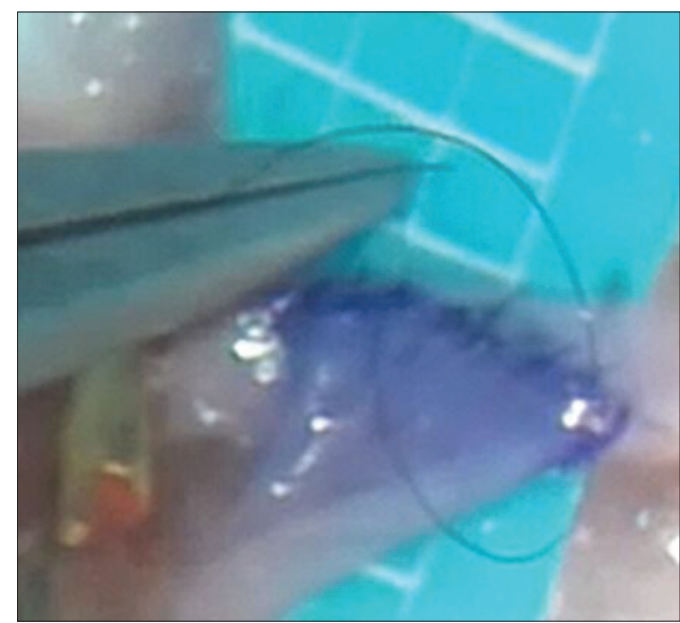

Figure 3:When holding the end of the thread to cut the remainder of the thread, keep the thread between the grasp of the forceps. In this manner, the thread is under your control and it is easier to start the second knot

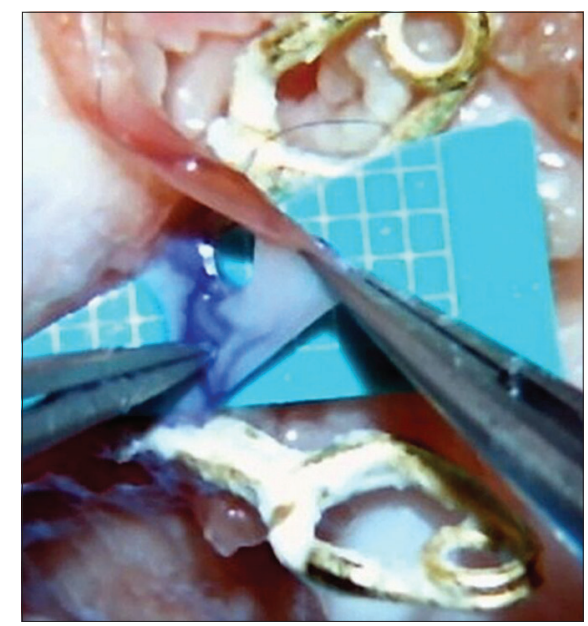

Figure 4: Putting the contralateral edge of the donor vessel inside the lumen of the recipient vessel makes it easier to avoid penetrating the contralateral edge of the recipient vessel

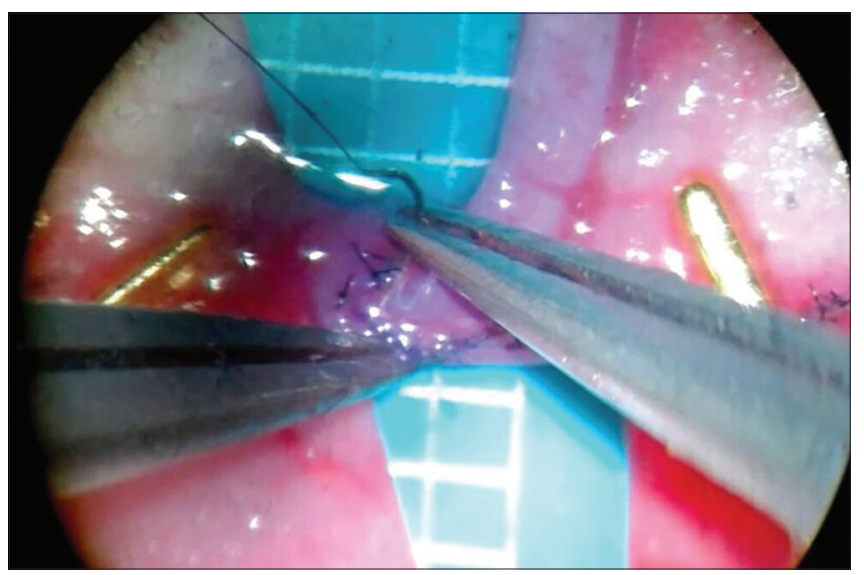

Figure 5:A clear surgical field is important. Irrigate when necessary but too much irrigation may work against you blurring the microscope view

suitable at this point. Continue with this stage for a minimum of 1 hour a day for 3 months 


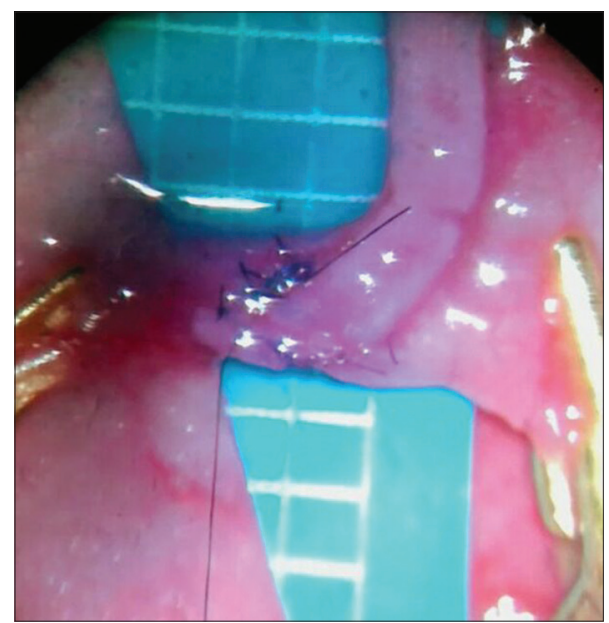

Figure 6: After making the knot, keep the thread end as short as possible to facilitate knotting.This will speed up knotting by avoiding unnecessary movements

- Focus on avoiding unnecessary movements. Count the time of every second bypass performed for 3 months. Start video recording to identify unnecessary movements. Use 10-0 thread

- Perform bypasses with a ticking clock for every second. Focus on speed. Record bypasses and preferably have some "audience" looking at you. Work against the clock

- Continuous training. After you have mastered the basics of microsuturing maintaining the ability is of paramount importance. Train at least 1 hour two times a week continuously. In our experience, even 1 month of absence from microsuturing sets you back

- Several neurosurgeons go to the laboratory to rehearse microsuturing on the night before the actual surgery (or in the morning of the surgery).

\section{CONCLUSION}

Neurosurgical bypass surgery is the art of excellent eye-hand coordination, fine motor skills, and micro-suturing knowledge. Training without studying the principle and surgical details could lead to frustrating result. We have summarized the experience of experienced vascular neurosurgeons to provide practical tricks and tips in this paper. All points have been tested in the laboratory setting and in real surgery for an extended period of time. We believe that bypass-training is a critical part of neurosurgical training and not only for a selected few. Diligent and meticulous training can enable every neurosurgeon to tame the art of bypass neurosurgery.
Financial support and sponsorship

Nil.

\section{Conflicts of interest}

There are no conflicts of interest.

\section{REFERENCES}

I. Abla AA, Lawton MT. Revascularization for unclippable posterior inferior cerebellar artery aneurysms: Extracranial-intracranial or intracranial-intracranial bypass? World Neurosurg 20I4;82:586-8.

2. Aboud ET, Krisht AF, O'Keeffe T, Nader R, Hassan M, Stevens CM, et al. Novel simulation for training trauma surgeons. J Trauma 201 I;7 I: I 484-90.

3. Alaraj A, Lemole MG, Finkle JH, Yudkowsky R, Wallace A, Luciano C, et al. Virtual reality training in neurosurgery: Review of current status and future applications. Surg Neurol Int 2012;2:52.

4. Chalouhi N, Zanaty M, Tjoumakaris S, Manasseh P, Hasan D, Bulsara KR, et al. Preparedness of neurosurgery graduates for neuroendovascular fellowship: A national survey of fellowship programs. J Neurosurg 2015;123:1 I I3-9.

5. Chowdhry SA, Spetzler RF. Genealogy of training in vascular neurosurgery. Neurosurgery 2014;74(Suppl I):SI 98-203.

6. Eivazi S, Hafez A, Fuhl W, Afkari H, Kasneci E, Lehecka M, et al. Optimal eye movement strategies: A comparison of neurosurgeons gaze patterns when using a surgical microscope. Acta Neurochir 2017;159:959-66.

7. Ezra DG, Aggarwal R, Michaelides M, Okhravi N, Verma S, Benjamin L, et al. Skills acquisition and assessment after a microsurgical skills course for ophthalmology residents. Ophthalmology 2009; I 16:257-62.

8. Harada K, Morita A, Minakawa Y, Baek YM, Sora S, Sugita N, et al. Assessing Microneurosurgical Skill with Medico-Engineering Technology. World Neurosurg 20I5;84:964-7I.

9. Harbaugh RE, Agarwal A. Training residents in endovascular neurosurgery. Neurosurgery 2006;59:S277-8I; discussion S273-2I3.

10. Harnof S, Hadani M, Ziv A, Berkenstadt H. Simulation-based interpersonal communication skills training for neurosurgical residents. Israel Med Assoc J 2013;15:489-92.

II. Hooten KG, Lister JR, Lombard G, Lizdas DE, Lampotang S, Rajon DA, et al. Mixed reality ventriculostomy simulation: Experience in neurosurgical residency. Neurosurgery 20 I4; I0(Suppl 4):576-8I; discussion 58I.

12. Korja M, Lehto H, Juvela S, Kaprio J. Incidence of subarachnoid hemorrhage is decreasing together with decreasing smoking rates. Neurology 2016;87: $1118-23$.

13. Liu JK, Kan P, Karwande SV, Couldwell WT. Conduits for cerebrovascular bypass and lessons learned from the cardiovascular experience. Neurosurg Focus 2003; 14:e3.

14. Regelsberger J, Heese O, Horn P, Kirsch M, Eicker S, Sabel M, et al. Training microneurosurgery-four years experiences with an in vivo model. Cent Eur Neurosurg 201 I;72:192-5.

15. Russell SM, Post N, Jafar JJ. Revascularizing the upper basilar circulation with saphenous vein grafts: Operative technique and lessons learned. Surg Neurol 2006;66:285-97.

16. Sarkiss CA, Philemond S, Lee J, Sobotka S, Holloway TD, Moore M, et al. Neurosurgical Skills Assessment: Measuring Technical Proficiency in Neurosurgery Residents through Intraoperative Video Evaluations. World Neurosurg 2016;89:1-8.

17. Schirmer CM, Mocco J, Elder JB. Evolving virtual reality simulation in neurosurgery. Neurosurgery 2013;73(Suppl I):127-37.

18. van der Zwan A. "How I Do It:” Non-occlusive High Flow Bypass Surgery. Acta Neurochir Suppl 2014;1 19:7I-6.

19. Yadav YR, Parihar V, Ratre S, Kher Y, Iqbal M. Microneurosurgical Skills Training. J Neurol Surg 2016;77:146-54. 